\title{
RATIONAL HOMOTOPY OF THE SPACE OF SECTIONS OF A NILPOTENT BUNDLE
}

\author{
BY \\ ANDRE HAEFLIGER
}

\begin{abstract}
We show that an algebraic construction proposed by Sullivan is indeed a model for the rational homotopy type of the space of sections of a nilpotent bundle.
\end{abstract}

In his paper L'homologie des espaces fonctionnels, $\mathrm{R}$. Thom studied the homotopy type of the space $F_{f}^{X}$ of continuous maps of $X$ into $F$ homotopic to a given map $f$.

Starting from a Postnikov decomposition of $F$, he built the functional space $F_{f} X$ step by step. He also indicated how one could construct a differential graded algebra describing the rational homotopy type of $F_{f}^{X}$.

Later on, Sullivan gave an algebraic model which mirrors this construction in terms of a DG-algebra representing $X$ and the minimal model of $F$.

The aim of this paper is to show, following the method of Thom, that the model of Sullivan is indeed a model for the functional space under suitable restrictions.

As in [3], we consider the slightly more general problem of the determination of the rational homotopy type of the space of sections $\Gamma_{s}$ of a nilpotent fiber space $p$ : $Y \rightarrow X$ homotopic to a given section $s$.

In $\S 1$ we explain Thom's geometric construction. In $\$ 2$ we describe an algebraic model for an abelian Galois covering of a nilpotent space. In $\S 3$ we show how the model of Sullivan fits with the geometry.

I thank the referee for many improvements of this paper.

1. A Postnikov factorization of the space of sections.

1.1. Let $G$ be a finitely generated abelian group and let $X$ be a path connected space whose cohomology groups $H^{k}(X ; G)$ are finitely generated for each $k$.

To avoid difficulties with the topologies (cf. [4]), we can work in the category of simplicial sets.

Proposition (Thом [4]). The space $K(G, m)^{X}$ of continuous maps of $X$ in the Eilenberg-Mac Lane complex $K(G, m)$ is homotopically equivalent to the product $\prod_{i=0}^{m} K_{i}$ of the Eilenberg-Mac Lane spaces $K_{i}=K\left(H^{m-i}(X ; G), i\right)$.

More precisely, let $\chi \in H^{m}(K(G ; m) ; G)$ be the fundamental class of $K(G ; m)$. If

$$
e: K(G, m)^{X} \times X \rightarrow K(G, m)
$$

Received by the editors September 18, 1979 and, in revised form, May 16, 1981. 1980 Mathematics Subject Classification. Primary 55P15, 55R05. 
is the evaluation map, we can write $e^{*}(\chi)$ uniquely as $\Sigma \chi_{i}$ where $\chi_{i} \in$ $H^{i}\left(K(G, m)^{X} ; H^{m-i}(X ; G)\right)$. Then the projection $K(G ; m)^{X} \rightarrow K_{i}$ is determined by the cohomology class $\chi_{i}$.

Proof. As $K(G ; m)$ is a Hopf space, all the connected components of $K(G, m)^{X}$ have the same homotopy type as the connected component $K(G, m)_{0}^{X}$ of the constant map. They are in bijection with $H^{m}(X ; G)=K\left(H^{m}(X ; G), 0\right)$.

If $f: S^{i} \times X \rightarrow K(G, m)$ represents an element of $\pi_{i}\left(K(G, m)_{0}^{X}\right)$, then its homotopy class is characterized by

$$
f^{*}(\chi) \in H^{0}\left(S^{i} ; Z\right) \otimes H^{m}(X ; G)+H^{i}\left(S^{i} ; Z\right) \otimes H^{m-i}(X ; G) .
$$

The first component of $f^{*}(\chi)$ vanishes because $f$ restricted to $S^{i} \times\{x\}$ is homotopic to a constant map. The second component is of the form $s \otimes u_{f}$, where $s$ is the canonical generator of $H^{i}\left(S^{i} ; \mathbf{Z}\right)$, and $u_{f} \in H^{m-i}(X ; G)$.

It is easy to see that the map $f \mapsto u_{f}$ induces an isomorphism of $\pi_{i}\left(K(G ; m)_{0}^{X}\right)$ on $H^{m-i}(X ; G)=\pi_{i}\left(\Pi K_{j}\right)$ and that the map $K(G ; m)^{X} \rightarrow \Pi K_{j}$ described above induces an isomorphism on homotopy groups, so is a homotopy equivalence.

1.2. Consequence. Let $Z$ be a topological space and let $f: Z \times X \rightarrow K(G ; m)$ be a continuous map. It gives a map $\varphi: Z \rightarrow K(G, m)^{X}$; its composition with the projection on $K\left(H^{m-i}(X ; G), i\right)$ will be denoted by $\varphi_{i}$. Then the homotopy class of $\varphi_{i}$ is determined by the component of

$$
f^{*}(\chi) \in H^{m}(Z \times X ; G)
$$

in $H^{i}\left(Z ; H^{m-i}(X ; G)\right)$.

It follows that the map induced by $\varphi_{i}$ on the rational cohomology can be described as follows.

Let $V=\operatorname{Hom}(G, Q)=H^{m}(K(G, m) ; Q)$. Then

$$
H^{i}\left(K\left(H^{m-i}(X ; G), i\right) ; Q\right) \approx H_{m-i}(X ; Q) \otimes V,
$$

where $H_{m-i}(X ; Q)$ is identified with the dual of $H^{m-i}(X ; Q)$.

The homomorphism $\varphi_{i}^{*}: H^{i}\left(K\left(H^{m-i}(X ; G), i\right), Q\right) \rightarrow H^{i}(Z ; Q)$ is given by

$$
\varphi_{i}^{*}\left(a^{\prime} \otimes v\right)=a^{\prime} \cap f^{*}(v),
$$

where $a^{\prime} \in H_{m-i}(X ; Q), v \in V$ and $a^{\prime} \cap(a \otimes b)=a^{\prime}(a) b$, for $a \otimes b \in H^{*}(X ; Q)$ $\otimes H^{*}(Z ; Q) \approx H^{*}(Z \times X ; Q)$.

1.3. Space of sections of a nilpotent bundle. Let $p: Y \rightarrow X$ be a bundle which admits a Moore-Postnikov factorization (in the sense of Spanier [2,pp. 437-444]). This means that the map $p$ is, up to homotopy equivalence, the composition of a possibly infinite sequence of fibrations

$$
X=Y_{0} \stackrel{p_{1}}{\leftarrow} Y_{1} \stackrel{p_{2}}{\leftarrow} Y_{2} \leftarrow \cdots,
$$

where $p_{r}: Y_{r} \rightarrow Y_{r-1}$ is a principal $K\left(G_{r}, n_{r}\right)$-bundle, $n_{r} \geqslant 1$. Also the sequence converges, i.e. for each positive integer $k$, then $n_{r}>k$ for $r$ large enough. We also assume $G_{r}$ abelian. Such a bundle will be called nilpotent.

We assume that the integral homology of $X$ is finitely generated (in particular $H_{j}(X, \mathbf{Z})=0$ for $j$ large) and that each $G_{r}$ is finitely generated. 
We want to describe the space $\Gamma_{s}$ of continuous sections of $p: Y \rightarrow X$ which are homotopic to a given section $s$.

From $s$, we obtain a sequence $s_{r}: X \rightarrow Y_{s}$ of compatible sections of $Y_{r} \rightarrow X$, i.e. $s_{r-1}=p_{r} s_{r}$. The principal bundle $p_{r}: Y_{r} \rightarrow Y_{r-1}$ is induced from the path space bundle $\pi: P \rightarrow K\left(G_{r}, n_{r}+1\right)$ by a map $c_{r-1}: Y_{r-1} \rightarrow K\left(G_{r}, n_{r}+1\right)$. We can assume that $c_{r-1} s_{r-1}$ maps $X$ on the base point.

Note that $P^{X}$ is isomorphic to the path space of $K\left(G_{r}, n_{r}+1\right)_{0}^{X}$, the space of maps of $X$ in $K\left(G_{r}, n_{r}+1\right)$ homotopic to a constant. The fiber above the constant map on the base point is $K\left(G_{r}, n_{r}\right)^{X}$, where $K\left(G_{r}, n_{r}\right)$ is the fiber of $P$ above the base point.

Let $\Gamma_{r}$ be the space of sections of the bundle $Y_{r} \rightarrow X$ which are homotopic to $s_{r}$. Let $q_{r}: \Gamma_{r} \rightarrow \Gamma_{r-1}$ be the map associating to a section $\sigma$ the section $p_{r} \cdot \sigma$.

Proposition. $\Gamma_{s}$ is homotopically equivalent to the limit of the convergent sequence of principal fibrations

$$
\Gamma_{1} \leftarrow \Gamma_{2} \leftarrow \cdots .
$$

Let $e_{r-1}: \Gamma_{r-1} \times X \rightarrow Y_{r-1}$ be the evaluation map $e_{r-1}(\sigma, x)=\sigma(x)$. The bundle $q_{r}: \Gamma_{r} \rightarrow \Gamma_{r-1}$ is the connected component of $s_{r}$ in the principal $K\left(G_{r}, n_{r}\right)^{X}$-bundle classified by the map $\bar{c}_{r-1}: \Gamma_{r-1} \rightarrow K\left(G_{r}, n_{r}+1\right)_{0}^{X}$ corresponding to $c_{r-1} \circ e_{r-1}$.

Indeed let $\hat{\Gamma}_{r}$ be the space of sections of $Y_{r} \rightarrow X$ projecting on a section of $Y_{r-1}$ homotopic to $s_{r-1}$. An element of $\hat{\Gamma}_{r}$ is given by a pair $(f, g)$ where $f: X \rightarrow P$ and $g$ : $X \rightarrow Y_{r-1}$, with $g \in \Gamma_{r-1}$ and $\pi \circ f=c_{r-1} \circ g$. This amounts to saying that $\hat{\Gamma}_{r}$ is the bundle over $\Gamma_{r-1}$ induced by $\bar{c}_{r-1}$ from $P^{X}$.

$\Gamma_{r}$ is just the connected component of $s_{r}$ in $\hat{\Gamma}_{r}$. The fiber of $\Gamma_{r} \rightarrow \Gamma_{r-1}$ is isomorphic to

$$
K\left(G_{r}, n_{r}\right)_{0}^{X} \times G^{\prime}
$$

where $G^{\prime} \subset H^{n_{r}}\left(X ; G_{r}\right)$ is the image of $\pi_{1}\left(\Gamma_{r}\right)$ by the homomorphism induced on $\pi_{1}$ by $\bar{c}_{r-1}$;

$$
\lambda: \pi_{1}\left(\Gamma_{r-1}\right) \rightarrow \pi_{1}\left(K\left(G_{r}, n_{r}+1\right)_{0}^{X}\right)=H^{n_{r}}(X ; G) .
$$

Indeed, let $\tilde{\Gamma}_{r-1} \rightarrow \Gamma_{r-1}$ be the covering whose fibers are the set of connected components of the fibers of $\Gamma_{r} \rightarrow \Gamma_{r-1}$. It is a Galois covering with group $G^{\prime}$. The fiber $\Gamma_{0}$ of $\Gamma_{r} \rightarrow \tilde{\Gamma}_{r-1}$ above the projection of $s_{r}$ is the set of sections of $Y_{r} \rightarrow X$ projecting on $s_{r-1}$ and homotopic to $s_{r}$ by a homotopy whose projection in $\Gamma_{r-1}$ is just the trivial path. So $\Gamma_{0}$ is canonically isomorphic to $K\left(G_{r}, n_{r}\right)_{0}^{X}$, because the bundle induced by $s_{r-1}$ from $Y_{r} \rightarrow Y_{r-1}$ is canonically isomorphic to $X \times K\left(G_{r}, n_{r}\right)$ (we have assumed that $c_{r-1} \circ s_{r-1}$ is the constant map).

\section{Model for a Galois covering of a nilpotent space.}

2.1. Notations. All DG-algebras $A$ (differential graded) will be defined over the field $Q$ of rationals, commutative in the graded sense and positively graded $\left(A^{q}=0\right.$ for $q<0$ ), unless otherwise specified.

A morphism $A \rightarrow B$ of DG-algebras is a weak equivalence, abbreviated w.e., if it induces an isomorphism $H(A) \rightarrow H(B)$ in cohomology. 
If $A$ is a DG-algebra and $V$ a graded vector space, then $A(V)$ will be a DG-algebra which is, as a graded algebra, the tensor product of $A$ with the symmetric algebra (in the graded sense) over $V$, with a differential $d$ extending the given differential on $A \subset A(V)$. We identify $V$ to the vector subspace $1 \otimes V$ in $A(V)$.

A model for a space $X$ is a DG-algebra $A$ together with a w.e. $\alpha: A \rightarrow \Omega^{*}(X)$, where $\Omega^{*}(X)$ denotes the DG-algebra of $Q$-polynomial forms on the singular complex of $X$.

For instance, let $G$ be an abelian group such that $V=\operatorname{Hom}(G ; Q)$ is a finite dimensional vector space over $Q$. Consider $V$ as a graded vector space homogeneous of degree $m$. Then the algebra $Q(V)$ of polynomial or alternate forms on $V$ according to the parity of $m$, with the zero differential, is a model for the Eilenberg-Mac Lane complex $K(G, m), m>0$.

We shall use repeatedly the following fact which follows easily from Grivel [1] (see also S. Halperin [5]).

Proposition. Let $X_{2} \rightarrow X_{1}$ be a principal abelian fibration whose fiber $X_{0}$ is a product of connected Eilenberg-Mac Lane complexes. Let $\varphi_{1}: A \rightarrow \Omega^{*}\left(X_{1}\right)$ be a morphism of augmented DG-algebras which is a model for $X_{1}$. Let $A(V)$ be a DG-algebra such that $d V \subset A$. Assume we have a morphism $\varphi_{2}: A(V) \rightarrow \Omega^{*}\left(X_{2}\right)$ such that the diagram

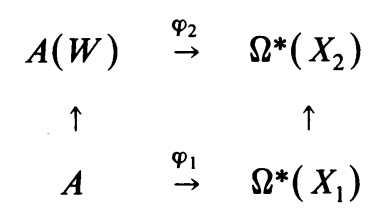

commutes.

Let $\varphi_{0}: Q(V) \rightarrow \Omega^{*}\left(X_{0}\right)$ be the induced morphism on the fiber. Then $\varphi_{2}\left(\right.$ resp. $\left.\varphi_{0}\right)$ is a w.e. if $\varphi_{0}\left(\right.$ resp. $\left.\varphi_{2}\right)$ is a w.e.d.

2.2. Abelian Galois covering of a nilpotent space. Let $X$ be a connected nilpotent space (i.e. $X$ considered as a bundle over a point is nilpotent as in 1.3). We assume that the homotopy groups $\pi_{i}(X), i>1$, are such that $\pi_{i}(X) \otimes Q$ are finite dimensional vector spaces, and the same property for the successive quotients in the lower central series of the nilpotent group $\pi_{1}(X)$.

Let $p: \tilde{X} \rightarrow X$ be a Galois covering with abelian Galois group $G$ such that $G \otimes Q$ is finite dimensional. It is classified by a surjective homomorphism

$$
\lambda: \pi_{1}(X) \rightarrow G .
$$

We consider $V=\operatorname{Hom}(G, Q)$ as a graded vector space homogeneous of degree 0 .

Let $\alpha: A \rightarrow \Omega^{*}(X)$ be a model for $X$. There is an injective linear map $h$ of $V$ in the cocycles of degree 1 of $A$ such that, when we pass to cohomology, $h$ is the dual $\lambda^{*}$ : $V \rightarrow \operatorname{Hom}\left(\pi_{1}(X), Q\right)=H^{1}(X ; Q)$ of $\lambda$. Note that the kernel of $p^{*}: H^{1}(X ; Q) \rightarrow$ $H^{1}(\tilde{X} ; Q)$ is the image of $\lambda^{*}$. 
On $A(V)$, the algebra of polynomials on $V$ with coefficients in $A$, consider the differential $d$ extending the differential of $A$ and such that $d(v)=h(v)$ for $v \in V$.

PROPOSITION. $A(V)$ is a model of $\tilde{X}$. More precisely, any morphism $\tilde{\alpha}: A(V) \rightarrow \Omega^{*}(\tilde{X})$ extending $\alpha$ is a model.

Proof. Consider a minimal Postnikov tower of $X$,

$$
X_{1} \leftarrow X_{2} \leftarrow X_{r-1} \leftarrow \cdots \leftarrow X_{r} \leftarrow \cdots \leftarrow X^{\prime},
$$

where each map is a principal abelian fibration, $X_{1}$ being $K\left(\pi_{1}(X) /\left[\pi_{1}(X), \pi_{1}(X)\right], 1\right)$ and $X^{\prime}=\lim _{\leftarrow} X_{r}$ being homotopy equivalent to $X$ by a map $X \rightarrow X^{\prime}$.

Let $B=Q(W)$ be a minimal model for $X$ (cf. [3]) reflecting the above decomposition: there is a filtration $W_{1} \subset W_{2} \subset \cdots \subset W$ such that $Q\left(W_{r}\right)$ is a DG-subalgebra of $Q\left(W_{r+1}\right)$ with $d W_{r+1} \subset Q\left(W_{r}\right)$, and $W_{1}=\operatorname{Hom}\left(\pi_{1}(X), Q\right)=H^{1}(X ; Q)$. There is also a morphism $Q(W) \stackrel{\varphi}{\rightarrow} \Omega^{*}\left(X^{\prime}\right)$ which is a weak equivalence and whose restriction $\varphi_{r}$ to $Q\left(W_{r}\right)$ gives a model $Q\left(W_{r}\right) \rightarrow \Omega^{*}\left(X_{r}\right)$ of $X_{r}$.

Consider the exact sequence

$$
0 \rightarrow N \rightarrow \pi_{1}(X) /\left[\pi_{1}(X), \pi_{1}(X)\right] \rightarrow G \rightarrow 0
$$

given by $\lambda$. It induces a fibration

$$
X_{1}=K\left(\pi_{1}(X) /\left[\pi_{1}(X), \pi_{1}(X)\right], 1\right) \rightarrow K(G, 1)
$$

with fiber $K(N, 1)$.

So we get a fibration $X^{\prime} \rightarrow K(G, 1)$ by composition $X^{\prime} \rightarrow X_{1} \rightarrow K(G, 1)$. The inclusion of its fiber $\tilde{X}^{\prime}$ in $X^{\prime}$ is homotopically equivalent to $p: \tilde{X} \rightarrow X$.

Let $W_{0} \subset W_{1}$ be the image of $V$ by $\lambda^{*}: V \rightarrow H^{1}(X ; Q)=W_{1}$. Choose $\varphi_{1}$ so that its restriction to $Q\left(W_{0}\right)$ is a model $Q\left(W_{0}\right) \rightarrow \Omega^{*}(K(G, 1))$. Let $B /\left(W_{0}\right)$ be the DG-algebra quotient of $B=Q(W)$ by the ideal generated by $W_{0}$. The composition of $B \rightarrow \Omega^{*}\left(X^{\prime}\right)$ with the restriction to the fiber $\tilde{X}^{\prime}$ vanishes on $W_{0}$. So we get a morphism $B /\left(W_{0}\right)$ in $\Omega^{*}\left(\tilde{X}^{\prime}\right)$ which is a model (in fact a minimal model) as follows from repeated applications of the proposition in 2.1.

On $B(V)$ define a differential $d$ as above by $d v=\lambda^{*} v, v \in V$. The map $B(V) \rightarrow$ $B /\left(W_{0}\right)$ obtained by mapping $V$ on 0 and taking the quotient on $B$ induces an isomorphism in cohomology (this can be proved by induction on $r$, using the filtration of $W$ by the $W_{r}$ ).

So we can get a homotopy commutative diagram

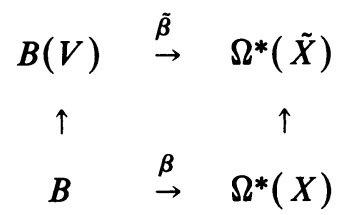

where the horizontal maps are w.e. In fact, we can assume the diagram commutative, because $B$ is a free nilpotent algebra.

Let $\alpha: A \rightarrow \Omega^{*}(X)$ be a model for $X$ and $\tilde{\alpha}: A(V) \rightarrow \Omega^{*}(\tilde{X})$ be a morphism extending $\alpha$ (such a morphism exists because $p^{*} \lambda^{*}=0$ ). As $B$ is the minimal model 
of $A$, there is a w.e. $f: B \rightarrow A$ extending to a morphism $f: B(V) \rightarrow A(V)$. It is easy to check that $f$ also induces an isomorphism in cohomology (filter by the degree of the polynomials in $V$ and use induction).

After changing $\beta$ by a homotopy, we can assume that $\alpha f=\beta$. Now $\tilde{\alpha} \tilde{f}$ and $\tilde{\beta}$ differs only by a map in the constant functions in $\Omega^{*}(\tilde{X})$, so they are homotopic. Hence $\alpha$ is also a weak equivalence.

REMARK. If $\lambda: \pi_{1}(X) \rightarrow G$ is not surjective, then the same result is valid if $\tilde{X}$ is replaced by one of the connected components of the Galois $G$-covering defined by $\lambda$, and $V$ replaced by its quotient $\bar{V}$ isomorphic to the image of $\lambda^{*}$.

\section{The model of Sullivan and the main theorem.}

3.1. The algebraic model of Sullivan. Let $p: Y \rightarrow X$ be a nilpotent bundle, i.e. admitting a Moore-Postnikov factorization through principal $K\left(G_{r}, n_{r}\right)$-fibrations as in 1.3. We assume that $V_{r}=\operatorname{Hom}\left(G_{r}, Q\right)$ is finite dimensional. It will be considered as a graded vector space homogeneous of degree $n_{r}$.

Let $\alpha: A \rightarrow \Omega^{*}(X)$ be a model for $X$. Then a model for $Y$, reflecting this Postnikov decomposition, will be of the form $A(V)$, where $V=\bigoplus V_{r}$ (cf. [3]). Suppose that $s$ : $X \rightarrow Y$ is a section. It gives a morphism $\sigma: A(V) \rightarrow A$ which is the identity on $A$. We can assume that $\sigma$ is zero on $V$.

Indeed if this is not the case, let $h$ be the $A$-algebra automorphism of $A(V)$ mapping $v$ on $v-\sigma(v)$; define on $A(V)$ a new differential $d^{\prime}$ such that $d h=h d^{\prime}$. Then $h$ is a DG-automorphism and $\sigma \circ h$ maps $V$ on zero.

$W e$ assume that $A$ is finite dimensional. Denote by $A$ the lower graded vector space whose $i$ th component $\underline{A}_{i}$ is $\operatorname{Hom}\left(A^{i}, Q\right)$. Let $\underline{A} \otimes V$ be the graded vector space whose component of degree $k$ is $\bigoplus_{-i+j=k} \underline{A}_{i} \otimes V^{j}$ (so in general we have components with negative degree).

There is a canonical $A$-algebra homomorphism

$$
\varepsilon^{\prime}: A(V) \rightarrow A \otimes Q(\underline{A} \otimes V)
$$

defined by $\varepsilon^{\prime}(a)=a \otimes 1, a^{\prime} \cap \varepsilon^{\prime}(v)=a^{\prime} \otimes v$ for each $a^{\prime} \in \underline{A}, v \in V$, where $a^{\prime} \cap$ $(a \otimes z)=a^{\prime}(a) z$, for $z \in Q(\underline{A} \otimes V)$.

In terms of an additive basis $a_{i}$ of $A$ and the dual basis $a_{j}^{\prime}$ of $\underline{A}$, then $\varepsilon^{\prime}(v)=\Sigma_{i} a_{i}$ $\otimes\left(a_{i}^{\prime} \otimes v\right)$.

On the algebra $Q(\underline{A} \otimes V)$, which is in general not positively graded, there is a unique differential $d$ such that $\varepsilon^{\prime}$ is a morphism of DG-algebras. The natural augmentation $Q(\underline{A} \otimes V) \rightarrow Q$ which is the identity on $Q$ and zero on $\underline{A} \otimes V$ commutes with the differentials.

The differential on $Q(\underline{A} \otimes V)$ induces on $\underline{A} \otimes V$ a differential $d_{0}$. Consider the quotient of $Q(\underline{A} \otimes V)$ by the ideal generated by elements of degree $\leqslant 0$ of $\underline{A} \otimes V$ and their differentials. It is isomorphic to the algebra $Q(W)$, where $W$ is the quotient of $\underline{A} \otimes V$ by elements of degree $\leqslant 0$ and their images by $d_{0}$. Note that $Q(W)$ is positively graded and $Q(W)^{0}=Q$.

From $\varepsilon^{\prime}$ we get a DG-map $\varepsilon: A(V) \rightarrow A \otimes Q(W)$. This is the model proposed by Sullivan for the evaluation map $e: X \times \Gamma_{s} \rightarrow Y$. It has the following universal property. Let $D$ be a DG-algebra such that $D^{0}=Q$, and let $f: A(V) \rightarrow A \otimes D$ be a 
morphism of augmented DG-algebras over $A$. Then there is a unique $\varphi: Q(W) \rightarrow D$ such that the diagram

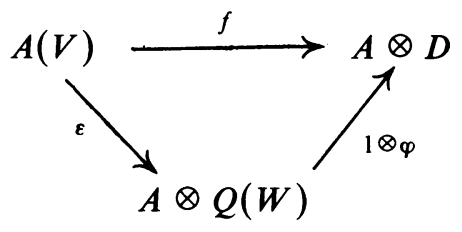

commutes.

3.2. TheOREM. Under the above assumptions ( $p: Y \rightarrow X$ a nilpotent bundle and $X$ admitting a finite dimensional model $),{ }^{1}$ the DG-algebra $Q(W)$ is a model for the space $\Gamma_{s}$ of sections of $p: Y \rightarrow X$ homotopic to a given section $s$. The morphism $\varepsilon$ is a model for the evaluation map $e$.

For the proof, we first show the theorem in the case of a trivial $K(G, m)$-bundle, using a model weakly equivalent to Sullivan's model. Then we assume by induction that the theorem is proved for the space $\Gamma_{r-1}$ of sections of the bundle $Y_{r-1} \rightarrow X$ in the tower of $p: Y \rightarrow X$ (cf. 1.3). We then construct in 3.5 an algebraic model for the bundle $\Gamma_{r} \rightarrow \Gamma_{r-1}$ (remember that the fiber is not connected in general) weakly equivalent to Sullivan's model, and show in 3.6 that it is indeed a model using $\$ 2$.

3.3. Case of a $K(G, m)$-trivial bundle. We assume that $Y=X \times K(G, m)$, with the section $s$ corresponding to the constant map of $X$ on the base point of $K(G, m)$.

In that case, $V=\operatorname{Hom}(G, Q)$ is homogeneous of degree $m$. The differential on $Q(\underline{A} \otimes V)$ is given by

$$
d\left(a^{\prime} \otimes v\right)= \pm \partial a^{\prime} \otimes v,
$$

where $\partial: \underline{A} \rightarrow \underline{A}$ is the transpose of $d$.

Let $W$ be the quotient of $\underline{A} \otimes V$ by the subspace of elements of degree $<0$ and cocycles in degree 0 .

Let $H_{*}(\underline{A})=\operatorname{Hom}\left(H^{*}(A), Q\right)=H_{*}(X ; Q)$. We can construct a linear injection $j$ : $H_{*}(A) \rightarrow \underline{A}$ mapping a homology class on a representative cycle.

Let $\bar{W}$ be the graded vector space defined by

$$
\begin{gathered}
\bar{W}^{k}=\bigoplus_{-i+j=k} H_{i}(\underline{A}) \otimes V^{j} \quad \text { for } k>0, \\
\bar{W}^{k}=0 \quad \text { for } k \leqslant 0 .
\end{gathered}
$$

$j$ gives an inclusion of $\bar{W}$ in $W$ and the corresponding inclusion $\bar{j}: Q(\bar{W}) \rightarrow Q(W)$ is a weak equivalence (the differential on $Q(\bar{W})$ is trivial).

There is a w.e.: $Q(V) \rightarrow \Omega^{*}(K(G, m))$ such that the induced map on cohomology gives the canonical isomorphism of $H^{m}(Q(V))=V$ on $H^{m}(K(G, m) ; Q)=$ $\operatorname{Hom}(G, Q)$.

\footnotetext{
${ }^{I} \mathrm{Cf}$. remark at the end for a less restrictive hypothesis.
} 
Let $\Gamma_{0}$ be the space $K(G, m)_{0}^{X}$ of maps of $X$ in $K(G, m)$ homotopic to the constant map on the base point, and let $e: X \times \Gamma_{0} \rightarrow X \times K(G, m)$ defined by $e(x, g)=$ $(x, g(x))$ be the evaluation map.

We can construct a homotopy commutative diagram

$$
\begin{array}{ccc}
\Omega^{*}\left(X \times \Gamma_{0}\right) & \stackrel{\Omega^{*}(e)}{\leftarrow} & \Omega^{*}(Y) \\
\uparrow & & \uparrow \\
A \otimes \Omega^{*}\left(\Gamma_{0}\right) & \stackrel{f}{\leftarrow} & A \otimes \mathbf{Q}(V)=A(V)
\end{array}
$$

where the vertical arrows are w.e., and $f$ is a morphism of DG-algebras over $A$ such that $f(v) \in A \otimes \Omega^{*}\left(\Gamma_{0}\right)^{+}$, where $\Omega^{*}\left(\Gamma_{0}\right)^{+}$is the kernel of the augmentation given by the base point.

There is a unique morphism $\varphi: Q(W) \rightarrow \Omega^{*}\left(\Gamma_{0}\right)$, mapping the class of $a^{\prime} \otimes v$ on $a^{\prime} \cap f(v)$, such that the diagram

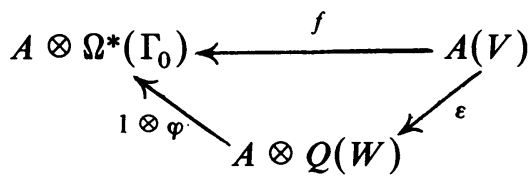

commutes, where $a^{\prime} \cap \varepsilon(v)$ is the class of $a^{\prime} \otimes v$.

To check that $\varphi$ (or $\varphi \circ \bar{j}$ ) is a weak equivalence, we pass to cohomology in the above diagram and use 1.2 which shows that $H^{*}\left(\Gamma_{0} ; Q\right)=Q(\bar{W})$ and gives the precise description of the evaluation map.

3.4 The induction hypothesis. For the general case of a nilpotent bundle, we take the notations of 1.3 .

To say that $A(V)$ is a model of $Y$ reflecting the factorization $X=Y_{0} \leftarrow Y_{1} \leftarrow Y_{2}$ $\leftarrow \cdots$ means that

(a) each $B_{r}=A\left(\oplus_{k<r} V_{k}\right)$ is a DG-subalgebra of $A(V)$, and $d V_{r} \subset B_{r-1}$, so that we have an increasing sequence of DG-subalgebras

$$
A=B_{0} \subset B_{1} \subset B_{2} \subset \cdots ;
$$

(b) for each $r$, we have a weak equivalence $\alpha_{r}: B_{r} \rightarrow \Omega^{*}\left(Y_{r}\right)$ such that the diagram

$$
\begin{array}{ccc}
B_{r}=B_{r-1}\left(V_{r}\right) & \stackrel{\alpha_{r}}{\rightarrow} & \Omega^{*}\left(Y_{r}\right) \\
\uparrow & & \uparrow \\
B_{r-1} & \stackrel{\alpha_{r-1}}{\rightarrow} & \Omega^{*}\left(Y_{r-1}\right)
\end{array}
$$

commutes and $\alpha_{r}$ induces a weak equivalence $Q\left(V_{r}\right) \rightarrow \Omega^{*}\left(K\left(G_{r}, n_{r}\right)\right)$ which in cohomology gives the canonical isomorphism $V_{r} \rightarrow H^{n_{r}}\left(K\left(G_{r}, n_{r}\right) ; Q\right)$; and

(c) we can choose $\alpha_{r}$ such that the diagram

$$
\begin{array}{ccc}
B_{r} & \stackrel{\alpha_{r}}{\rightarrow} & \Omega^{*}\left(Y_{r}\right) \\
\downarrow^{\sigma_{r}} & & \downarrow^{\Omega^{*}\left(s_{r}\right)} \\
A & \rightarrow & \Omega^{*}(X)
\end{array}
$$

commutes, where $\sigma_{r}$ is the restriction of $\sigma$ to $B_{r}$. 
Let $C_{r-1}$ be the Sullivan model for $\Gamma_{r-1}$. We assume by induction that we have a w.e. $\varphi_{r-1}: C_{r-1} \rightarrow \Omega^{*}\left(\Gamma_{r-1}\right)$ so that the diagram

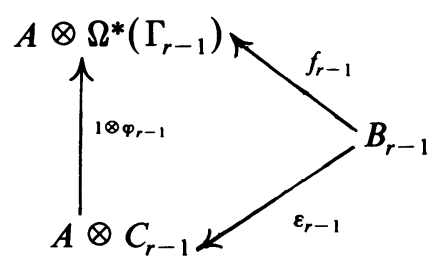

commutes, where $f_{r-1}$ is a model for the evaluation map $e_{r-1}: X \times \Gamma_{r-1} \rightarrow Y_{r-1}$. We also assume that $\sigma_{r-1}$, together with the augmentations in $C_{r-1}$ and $\Omega^{*}\left(\Gamma_{\mathrm{r}-1}\right)$ (the latter given by $s_{r-1}$ ) give morphisms of the diagram in $A$ so that everything is commutative.

3.5. Construction of an algebraic model. We construct as follows a model for the fibration $\Gamma_{r} \rightarrow \Gamma_{r-1}$.

There is an algebra homomorphism $\varepsilon_{r}^{\prime}$ such that

$$
\begin{array}{ccc}
A \otimes C_{r-1}\left(\underline{A} \otimes V_{r}\right) & \stackrel{\varepsilon_{r}^{\prime}}{\leftarrow} & B_{r-1}\left(V_{r}\right)=B_{r} \\
\uparrow & & \uparrow \\
A \otimes C_{r-1} & \stackrel{\varepsilon_{r-1}}{\leftarrow} & B_{r-1}
\end{array}
$$

commutes (compare with 3.1), defined by $a^{\prime} \cap \varepsilon_{r}^{\prime}(v)=a^{\prime} \otimes v$.

On $C_{r-1}\left(\underline{A} \otimes V_{r}\right)$, there is a unique differential such that $\varepsilon_{r}^{\prime}$ commutes with $d$;

$$
d\left(a^{\prime} \otimes v\right)= \pm \partial a^{\prime} \otimes v+ \pm a^{\prime} \cap \varepsilon_{r-1}(d v) .
$$

Let $C_{r-1}(U)$ be the quotient of $C_{r-1}\left(\underline{A} \otimes V_{r}\right)$ by the ideal generated by elements of $\underline{A} \otimes V_{r}$ of degree $<0$ and those elements of degree 0 whose boundary is in the ideal generated by elements of negative degree (this ideal is closed under $d$ and $H^{0}\left(C_{r-1}(U)\right)=Q$ because $\left.C_{r-1}^{0}=Q\right)$.

We get from $\varepsilon_{r}^{\prime}$ a morphism

$$
\varepsilon_{r}: B_{r-1}\left(V_{r}\right) \rightarrow A \otimes C_{r-1}(U)
$$

extending $\varepsilon_{r-1}$. We shall prove that this is a model for $e_{r}$.

$C_{r-1}(U)$ verifies the following universal property: let $D$ be a DG-algebra with an augmentation and such that $H^{0}(D)=Q$. Given a commutative diagram

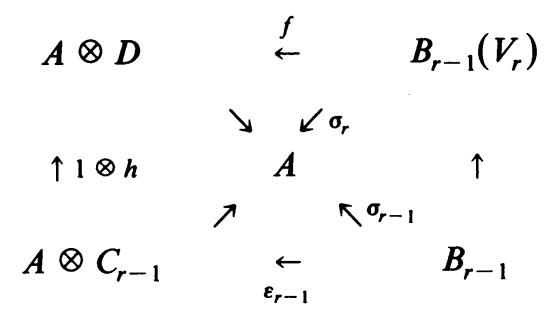

where the left-hand maps in $A$ are the tensor product of the identity on $A$ with the 
augmentation, there is a unique morphism $\varphi: C_{r-1}(U) \rightarrow D$ of augmented DG-algebras over $C_{r-1}$ such that

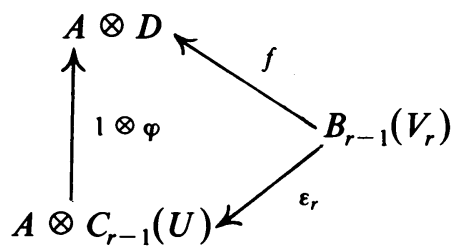

commutes. On the class $u$ of $a^{\prime} \otimes v, \varphi$ is given by

$$
\varphi(u)=a^{\prime} \cap f(v) \text {. }
$$

We get the Sullivan model $C_{r}$ by taking the quotient of $C_{r-1}(U)$ by the ideal generated by elements of $U$ of degree 0 and their differentials.

It will be sufficient to prove that $\varepsilon_{r}$ is a model for $e_{r}$, once we have checked that the quotient map $C_{r-1}(U) \rightarrow C_{r}$ is a weak equivalence.

$C_{r-1}$ is of the form $Q(S)$ with $S^{p}=0$ for $p \leqslant 0$. Hence the differential $d$ maps $U^{0}$ injectively in $U^{1} \oplus S^{1}$. The DG-algebra $C_{r-1}(U)$ is nilpotent, hence we can choose elements $x_{1}, \ldots, x_{k}$ of $U \oplus S$ so that $C_{r-1}(U)=Q\left(U^{0}, d U^{0}\right)\left(x_{1}, \ldots, x_{k}\right)$ and so that $d x_{i+1} \in Q\left(U^{0}, d U^{0}\right)\left(x_{1}, \ldots, x_{i}\right)$. By induction on $i$, the projection of $Q\left(U^{0}, d U^{0}\right)\left(x_{1}, \ldots, x_{i}\right)$ on its quotient by the ideal generated by $U^{0}$ and $d U^{0}$ is a w.e. When $i=k$ this is the projection of $C_{r-1}(U)$ on $C_{r}$.

3.6. Proof of the induction step. One can find a morphism $f_{r}$ which is a model for the evaluation map $e_{r}: X \times \Gamma_{r} \rightarrow Y_{r}$ and such that

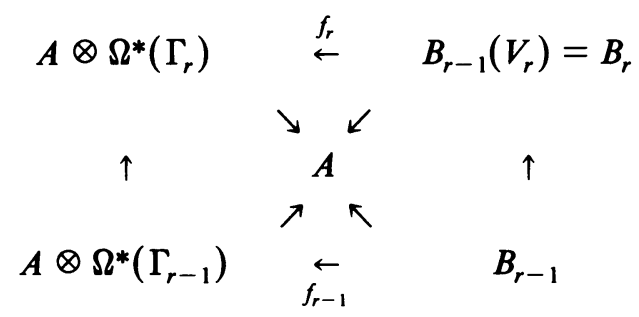

commutes.

By the universal property, we get a morphism

$$
\varphi_{r}: C_{r-1}(U) \rightarrow \Omega^{*}\left(\Gamma_{r}\right)
$$

such that the diagram

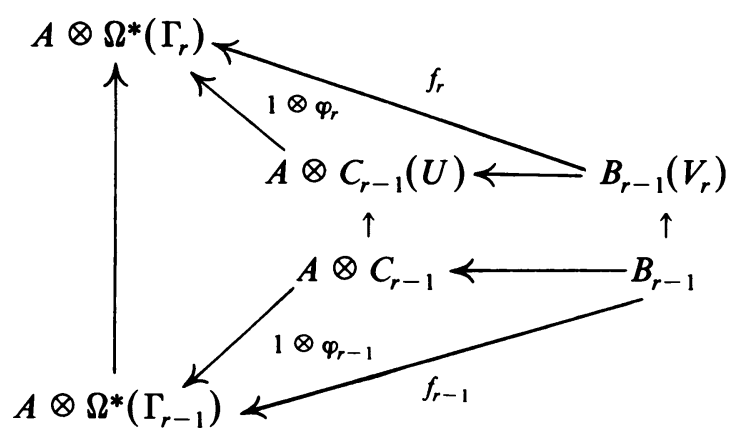

commutes. 
We have to show that $\varphi_{r}$ is a weak equivalence. For $u \in U, d u=d_{0} u+d_{1} u$, where $d_{0} u \in U$ and $d_{1} u \in C_{r-1}$ (cf. 3.5).

Let $\bar{U}^{0}$ be the kernel of $d_{0}: U^{0} \rightarrow U^{1}$. Then $C_{r-1}\left(\bar{U}^{0}\right)$ is a DG-subalgebra of $C_{r-1}(U)$ and $d$ maps $\bar{U}^{0}$ injectively into the 1-cocycles of $C_{r-1}$. When we pass to cohomology $d: \bar{U}^{0} \rightarrow H^{1}\left(C_{r-1}\right)=H^{1}\left(\Gamma_{r-1} ; Q\right)$ is still injective because $C_{r-1}^{0}=Q$. The image is generated by cohomology classes of elements of the form $a^{\prime} \cap \varepsilon_{r-1}(d v)$, where $v \in V_{r}, a^{\prime} \in \underline{A}_{n_{r}}$ with $\partial a^{\prime}=0$.

As in 1.3, we consider the factorization $\Gamma_{r} \rightarrow \tilde{\Gamma}_{r-1} \rightarrow \Gamma_{r-1}$. We get a commutative diagram

$$
\begin{array}{ccc}
C_{r-1}(U) & \stackrel{\varphi_{r}}{\rightarrow} & \Omega^{*}\left(\Gamma_{r}\right) \\
\uparrow & & \uparrow \\
C_{r-1}\left(\bar{U}^{0}\right) & \stackrel{\tilde{\varphi}_{r-1}}{\rightarrow} & \Omega^{*}\left(\tilde{\Gamma}_{r-1}\right) \\
\uparrow & & \uparrow \\
C_{r-1} & \stackrel{\varphi_{r-1}}{\rightarrow} & \Omega^{*}\left(\Gamma_{r-1}\right)
\end{array}
$$

because $\varepsilon_{r}$ maps elements of $\bar{U}^{0}$ on functions on the singular complex of $\Gamma_{r}$ whose differential comes from $\Omega^{*}\left(\Gamma_{r-1}\right)$, hence are constant on the connected components of the fibers of $\Gamma_{r} \rightarrow \Gamma_{r-1}$.

We claim that $\tilde{\varepsilon}_{r-1}$ is a weak equivalence. Indeed, $\tilde{\Gamma}_{r-1}$ is a connected component of the Galois covering defined by the homomorphism $\lambda: \pi_{1}\left(\Gamma_{r}\right) \rightarrow H^{n_{r}}\left(X, G_{r}\right)$ induced by $\bar{c}_{r-1}: \Gamma_{r-1} \rightarrow K\left(G_{r}, n_{r}+1\right)_{0}^{X}$ (cf. 1.3). Its dual gives a map

$$
\lambda^{*}: \operatorname{Hom}\left(H^{n_{r}}\left(X ; G_{r}\right), Q\right)=H_{n_{r}}(X ; Q) \otimes V_{r} \rightarrow H^{1}\left(\Gamma_{r-1} ; Q\right)
$$

whose image is isomorphic to $d \bar{U}^{0}$ by the remark above and 1.2. So we can apply the proposition and the last remark of 2.2 to deduce that $\tilde{\varepsilon}_{r-1}$ is a weak equivalence ( $\Gamma_{r-1}$ is a nilpotent space).

$C_{r-1}(U)$ can be considered as an algebraic bundle over $C_{r-1}\left(\bar{U}^{0}\right)$, with fiber $Q(W)=C_{r-1}(U) \otimes_{C_{r-1}\left(\bar{U}^{0}\right)} Q$ where $Q$ is considered as a $C_{r-1}\left(\bar{U}^{0}\right)$-module via the augmentation. The vector space $W$ is isomorphic to the quotient of $A \otimes V_{r}$ by the subspace of elements of degree $<0$ and $d_{0}$-cycles in degree 0 . We can express $C_{r-1}(U)$ as $C_{r-1}\left(\bar{U}^{0}\right) \otimes Q(W)$, where the differential of $w \in W$ has a component in $W$ and another one of degree at least 2 in $C_{r-1}$.

Recall that the fiber $\Gamma_{0}$ of $\Gamma_{r} \rightarrow \tilde{\Gamma}_{r-1}$ containing $s_{r}$ is isomorphic to $K\left(G_{r}, n_{r}\right)_{0}^{X}$, where $K\left(G_{r}, n_{r}\right)$ is the fiber of $Y_{r} \rightarrow Y_{r-1}$.

As $\tilde{\varphi}_{r-1}$ preserves the augmentation, we get a morphism $\varphi_{r}^{0}$ of the algebraic fiber $Q(W)$ in the DG-algebra of forms $\Omega^{*}\left(\Gamma_{0}\right)$ of the geometric fiber, and the diagram (1) gives the commutative diagram

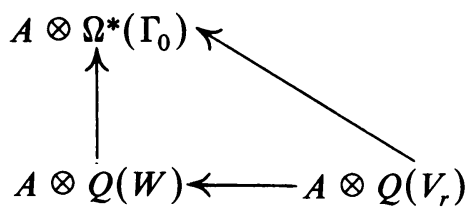

and we show as in 3.3 that $\varphi_{r}^{0}$ is a weak equivalence. 
As $\varphi_{r}$ induces a weak equivalence on the base and the fiber, we can apply the proposition in 2.1 to show that $\varphi_{r}$ is also a weak equivalence.

Consider the commutative diagram over $A$

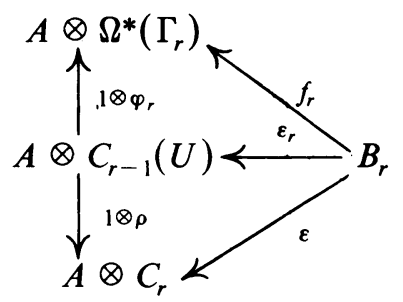

when $\rho: C_{r-1}(U) \rightarrow C_{r}$ is the w.e. described in 3.5 and $\varepsilon$ the morphism defined in 3.1.

As $\rho$ is a surjective w.e., then there is a w.e. $i: C_{r} \rightarrow C_{r-1}(U)$ such that $\rho \circ i=\mathrm{id}$. Then $\varphi=\varphi_{r} \circ i: C_{r} \rightarrow \Omega^{*}\left(\Gamma_{r}\right)$ is a w.e. and $f=(1 \otimes \varphi) \circ \varepsilon$ is homotopic over $A$ to $f_{r}$, hence is a model for the evaluation map $X \times \Gamma_{r} \rightarrow Y_{r}$.

REMARK. In the construction 3.1 of the Sullivan model, we only need to assume $A$ finite dimensional in each degree provided $V$ is finite dimensional, because the DG-algebra $Q(W)$ depends only on elements of $A$ whose degree is not more than the maximal degree of the elements in $V$ (same remark for 3.5).

Hence the main theorem 3.2 , with essentially the same proof, is valid under the hypothesis that either

(i) the rational homotopy of the fiber of the nilpotent bundle $p: Y \rightarrow X$ is finite dimensional and $X$ has a model $A$ which is finite dimensional in each degree, or

(ii) $X$ has a model $A$ which is finite dimensional in each degree and such that $\operatorname{dim} H^{*}(A)$ is finite.

Indeed in that case, we can prove the induction step 3.5 using $A$; to pass to the limit when $r$ tends to infinity, we can replace $A$ by a finite dimensional algebra.

\section{REFERENCES}

1. P. P. Grivel, Formes différentielles et suites spectrales, Ann. Inst. Fourier (Grenoble) 29 (1979).

2. E. H. Spanier, Algebraic topology, McGraw-Hill, New York, 1966.

3. D. Sullivan, Infinitesimal computation in topology, Inst. Hautes Études Sci. Publ. Math. 47 (1977), 269-331.

4. R. Thom, L'homologie des espaces fonctionnels, Colloq. Topol. Algébrique, Louvain, 1956, pp. 29-39.

5. S. Halperin, Lectures on minimal models, mimeographed notes, Lille.

Department of Mathematics, University of Geneva, CH-1211 Geneve 24, Switzerland 